\title{
ASPECTOS DE LA TÉCNICA ESCULTÓRICA IBÉRICA EN EL SIGLO V a. C.
}

\author{
IVÁN NEGUERUELA \\ Museo Arqueológico Nacional
}

\begin{abstract}
El estudio de las esculturas ibéricas de Porcuna ha permitido reconocer y reproducir algunas de las herramientas utilizadas por los escultores ibéricos: cincel de filo recto y curvo, taladro y lija.

The study of the Iberic sculptures from Porcuna has allowed to recognize and reproduce some of the tools utilized by the Iberians: right and curved chisels, drill and sandpaper.
\end{abstract}

Hasta la fecha han sido muy escasas las observaciones que se han producido en la bibliografía sobre un aspecto del estudio de la Plástica Ibérica tan importante como es el conocimiento de la técnica y del instrumental empleado en su ejecución. Por ello nos proponemos en estas páginas avanzar las primeras conclusiones obtenidas del estudio del Conjunto escultórico Ibérico del Cerillo Blanco de Porcuna (1).

Debido precisamente a esa falta de detenidos estudios al respecto carecemos de datos de referencia que nos permitan establecer generalizaciones o paralelos tanto técnicos cuanto cronológicos. La importancia que tiene el analizar los aspectos técnicos en el Conjunto del Cerillo Blanco radica en los siguientes puntos:

(1) NEGUERUELA: La escultura Ibérica del Cerrillo Blanco de Porcuna, Jaén. Estudio sobre su estructura interna, agrupamientos e interpretación. Madrid, 1990, con toda la bibliografía anterior.
1. Se dispone de un número dè esculturas y fragmentos muy elevado (1486) en los cuales se pueden conseguir suficientes observaciones y mediciones como para disminuir ampliamente los márgenes de error que se producirían trabajando con una sola escultura.

2. Entre las obras, las hay de entrego, exentas, en bulto redondo, grupos enlazados, altorelieves, caras vistas y ocultas, elementos arquitectónicos, etc, etc... es decir, un amplio abanico de ocasiones diferentes en las que poder analizar las distintas soluciones técnicas.

3. Las obras analizadas gozan de absoluta homogeneidad geográfica y posiblemente tambien cronológica. Todas provienen del yacimiento del $\mathrm{Ce}$ rrillo y fueron enterradas al mismo tiempo: en algún momento del siglo $\mathrm{V}$ a $\mathrm{C}$., si se quiere pecar de exceso de prudencia, $y$, con más probabilidad, en su primera mitad. Y aún si se piensa que hay diferentes momentos entre las obras, las últimas no pueden en modo alguno rebasar el citado siglo $\mathrm{V}$. 
4. Se trata en todos los casos de la misma piedra, lo que redundará en la homogeneidad de las observaciones.

Es decir, que al hacer los análisis sobre obras del mismo yacimiento, realizadas sobre el mismo tipo de piedra, con la misma cronología y en un número tan elevado, los datos que se extraigan, aún en el caso de ser incompletos, permitirán establecer un primer escalón fijo en nuestro conocimiento del desarrollo de aquella técnica escultórica. Estudios similares con materiales de

a) otras latitudes

b) otras cronologías

permitirán ir estableciendo el cuadro completo tanto por siglos cuanto por regiones peninsulares.

Los datos que a continuación se exponen como avance de nuestras ideas al respecto están basados en la observación, medición y cuantificación directa sobre el material. Hemos procedido a individualizar distintos tipos de huellas y posteriormente las que son iguales y pertenecen por tanto a la misma técnica utilizada en distintas esculturas las hemos medido sistemáticamente con el objetivo de reducir en lo posible los márgenes de error mediante el cotejo entre diversas obras a fin de conseguir el refrendo perseguido.

Esquemáticamente, el proceso, tal y como puede ser hoy documentado, seguía una serie de pasos desde el desbastado inicial a cincel hasta el pulido final. En este proceso es obvio que los instrumentos más evidentes son los que han dejado sus huellas en las partes que no han sido sometidas al tratamiento final de pulido y lijado, debido a que éste ha borrado los procesos anteriores.

Según todo ello, los resultados que hemos obtenido son los siguientes:

\section{TRATAMIENTO DE LAS BASAS}

La estructura básica de las basas está constituida por un prisma cuyas caras son:

a) plano inferior (la cara que da al suelo).

b) planos o caras verticales.

c) plano superior (la cara que sirve de suelo a la escultura).

d) chaflán (la transición entre el plano superior y los verticales).

Los tipos de bases que existen en el Cerrillo Blanco son:

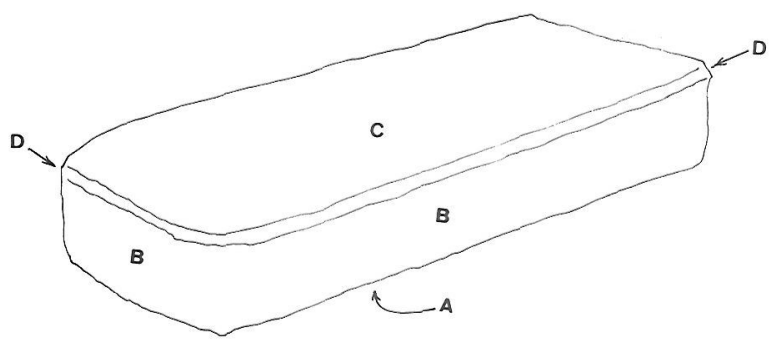

Fig. 1.- Estructura de una basa.

1. Basas para figuras o grupos en bulto redondo, con las caras verticales sin pulir

Sobre estas basas se sitúan figuras aisladas o grupos esculpidos en bulto redondo, con independencia de que tengan un punto de vista principal o varios. Se incluyen en este grupo las basas del Monumento de los Guerreros, la del Oferente con Cápridos, las de los grupos de 'machias' (gripomachia, leontomachia, lykomachia...), las de los novillos, etc... Es, de hecho, el grupo de basas más frecuente en el Cerrillo.

\subsection{Huellas de cinceles.}

En todos los casos, aparecen estas huellas en la cara inferior de las basas. También en todos los casos, aparecen en las caras verticales. En los chaflanes, más alisadas.

Las huellas de la cara que da al suelo, el plano inferior, tienen como objetivo buscar un plano suficientemente horizontal que sirva de apoyo al grupo escultórico. Por el contrario, las de las caras verticales manifiestan una intención muy expresa de obtener una superficie irregular, con desniveles y abultamientos que no se han querido uniformar. Sin embargo, existen variaciones: las rugosidades de las basas de los novillos son mucho mayores que las de

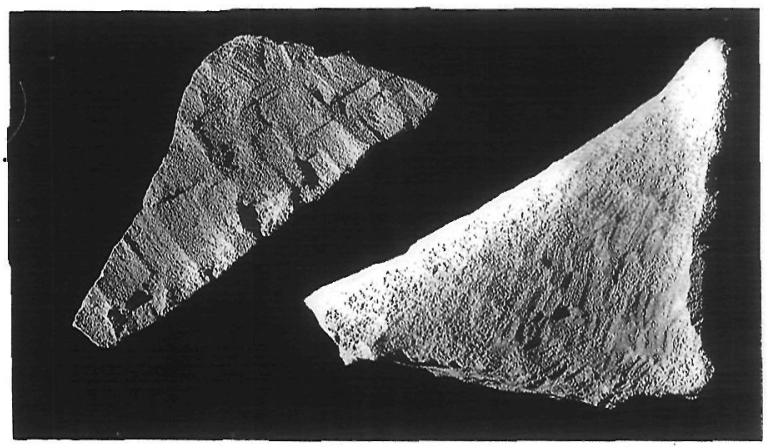

Lám. 1.- Fragmentos con huellas de cinceles diversos. 
los personajes o los grupos. En el caso de los chaflanes con los cuales se ha resuelto la unión de los planos verticales con el plano horizontal, el cincel se ha pasado en un ángulo muy cerrado en relación con la superficie de las piedras a fin de alisar este chaflán y que las transición entre el pulido plano superior y los rugosos planos verticales sea poco acusada.

\section{Basas para figuras exentas con las caras verti- cales bien pulidas..}

Son solamente dos en todo el conjunto del Cerrillo, la de una estatua sedente (González Navarrete, pp. 196 y 197) y la de una escultura no identificada, e inédita, en la que hay una pata de ave.

\subsection{Huellas de cinceles.}

En los dos casos, los planos inferiores son igual que en las basas comentadas anteriormente. Pero los planos verticales han sido muy bien alisados y pulidos, lo que ha borrado las huellas del rugoso cincelado que se observa en aquellas. En ambos casos, la transición entre los planos verticales y el plano superior horizontal se hace en ángulo recto en lugar de en chaflán como en las basas anteriores. De todo ello se colige que estas basas son completamente diferentes de todas las demás del Cerrillo.

\section{Basas de los altorelieves.}

Se integran en este grupo dos obras prácticamente completas y un pequeño fragmento de una tercera (G. Navarrete, Lamns. 129, 137, 198-38). La obra aquí no es exenta sino que se trata de esculturas adosadas en altorelieve a un plano vertical posterior, plano vertical del que también sale la basa.

\subsection{Huellas de cinceles.}

El bloque que sirve de fondo a las escenas presenta en su cara posterior (la que no se veía) numerosas huellas del cincel que se ha utilizado para desbastar. Por contra, las otras cuatro caras de estos fondos están bien alisados, pero no tanto como para haber borrado completamente el recuerdo del cincel.

Las basas siguen en su plano inferior la misma tónica que todas las demás, pero en sus caras verticales se ha omitido el alisado, el cual se ha sustitui- do por un cincelado más cuidadoso. Conviene resaltar que la basa del cazador de liebre presenta arista en la unión del plano vertical con el superior, mientras que en el caso de los pugilistas esta arista se ha sustituido por un chaflán.

\section{LOS FRAGMENTOS DE GOLAS}

Los restos, escasos, arquitectónicos que se conservan en el Cerrillo están constituidos por las golas que en otro lugar se comentan. Se trata de grandes bloques cuyas caras superior e inferior no iban vistas sino en contacto con otros elementos de la construcción de que se tratase. Lo mismo cabe para las varas verticales posterior y laterales (en el caso de que no se trate de un bloque en esquina). De modo que tan solo iba exenta la cara anterior en la cual se había esculpido la gola que define a estas piezas.

\section{Huellas de cincel.}

Todas las caras citadas, salvo las que iban vistas, presentan un aspecto muy similar al de los planos inferiores de las basas, es decir que han sido desbastadas y "alisadas" a base de cincel hasta obtener un plano suficientemente vertical $u$ horizontal como para poder contactar con limpieza con los bloques adyacentes. Las superficies de las caras vistas, es decir de las golas, han sido por el contrario bien pulidas (aún cuando en algunos casos se puedan observar aún huellas de los cinceles curvos con los que fueran talladas) y las uniones con las caras superior, inferior y laterales se hacen mediante una arista viva.
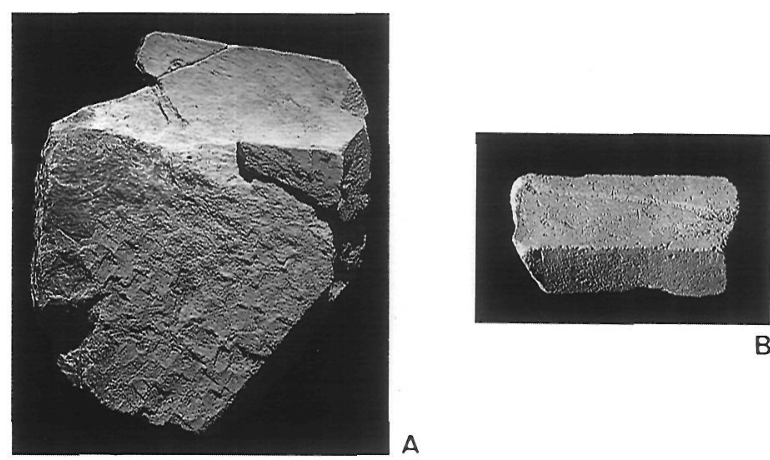

B

Lám. 2.- A. Gola arquitectónica. Huellas de cincel en la cara superior; acabado final en la cara lateral. B. Fragmento con acabado pulimentado. 


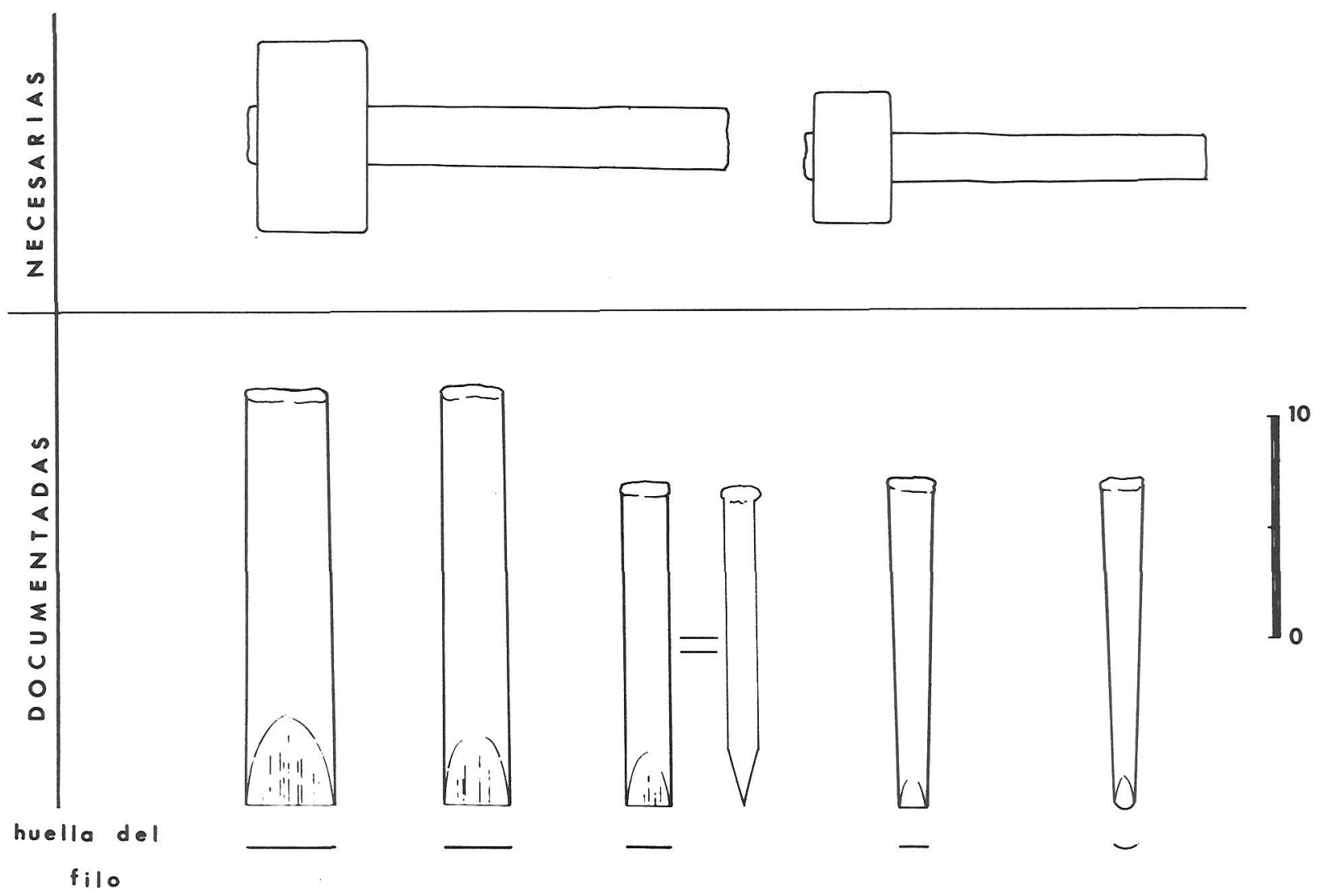

Fig. 2.- Tabla de herramientas.

\section{TIPOS DE CINCELES USADOS}

La toma de un puñado de medidas de las huellas que haya podido dejar, por ejemplo, un cincel, es insuficiente por cuanto el escultor, al dar golpes consecutivos al utensilio, está alterando constantemente las huellas anteriores de anchura que nos permitirían medir con precisión la boca del cincel. Y ello es tanto más así cuanto más exterior está ubicada dicha huella. De tal manera que tan solo las huellas que presentaran toda su anchura excavada en lo más interno de la superficie de la piedra podrían garantizarnos mediciones seguras. Con todo, hemos buscado exhaustivamente las huellas más representativas de cada anchura de boca, las que menos han sido alteradas por retoques posteriores. $\mathrm{Y}$ las hemos cotejado escultura a escultura para buscar el refrendo.

Según ello, podemos decir que se usó un cincel de boca ancha, de unos $4 \mathrm{~cm}$., para la tarea del desbastado de la obra. El filo del mismo era recto. Sus huellas son aún muy visibles y fácilmente mensurables en varias de las basas y partes ocultas de los elementos arquitectónicos y de los altorelieves.

Además, se usó el mismo instrumento con diferentes anchuras de boca; cuando menos, es posible detectar rastros de un cincel de $3 \mathrm{~cm}$. de boca; otro de $2 \mathrm{~cm}$., $\mathrm{y}$ aún otro que oscila entre $1,1 \mathrm{y}$ $1,4 \mathrm{~cm}$.

Estos cinceles se usaban de dos maneras. En unos casos se golpeaba con el instrumento casi adosado a la piedra, lo que permitía recorridos largos del filo sobre la superficie de la piedra, de tal modo que se conseguía con ello ir alisando bastante bien la citada superficie. Este modo se usó para realizar los chaflanes citados de las basas, y en otras partes en las que se pretendía dejar la obra a punto para su pulido posterior.

En otros casos, fundamentalmente en las caras verticales de las bases y posteriores de los altos relieves, el cincel se usaba abriendo su ángulo de incidencia con relación al plano de la superficie a es- 
culpir. Al ser mayor este ángulo, el cincel hacía menos recorrido pero penetraba más, de modo que dejaba un escalón al final de su recorrido (y dos escalones más en los laterales del mismo). Cuando la superficie resultante no requería la consecución de un plano especialmente recto, se usaba el cincel de boca mayor y con poco cuidado, como si se hubiera buscado expresamente conseguir un efecto de acusada rugosidad. El caso más claro de ello es el de las caras verticales de las basas de los novillos.

Pero si era necesario conseguir una superficie notoriamente recta ( $\sin$ que se necesitara llegar al estado de alisarla y pulirla) como, por ejemplo, en el caso de las superficies de apoyo y contacto de las golas arquitectónicas, entonces, con los mismos cinceles se procedía a ir suprimiendo el mayor número posible de rugosidades hasta obtener una superficie plana, para lo cual, inapelablemente hubo de ser utilizada una regla que sirviera de guía.

El hecho de que las basas presenten tantas rugosidades en sus planos verticales (más acusadas que en ningún otro caso en las basas de los novillos) nos lleva a pensar que es debido a que estas esculturas iban semienterradas en el suelo, y que estas paredes se dejaron así tanto por el hecho de que no iban a ser vistas cuanto para afianzar su agarre al suelo, al terreno colindante. Sin embargo aquellas obras que presentan unos planos verticales más cuidados no irían en esta situación. Ni obviamente tampoco las bases bien pulidas. Este esquema permite situar sobre el suelo a todas las estatuas de animales de las que nos ha llegado la basa. En todo caso, están estas basas tan limpias y sus aristas y rugosidades tan vivas que se diría que nunca se utilizaron.

En algunos casos quedan huellas de un cincel suavemente curvo ("curved chisel") de unos 9 milímetros de anchura en su boca. Se usó para afinar, en determinadas zonas, el desbastado que dejaban los instrumentos anteriores.

Otro tipo de huellas está constituido por planos alargados que se yuxtaponen entre sí mediante una arista de escasísimo relieve y sinuosa, como si con el filo de un cuchillo se hubiese ido igualando la rugosa superficie dejada por los cinceles más finos. El efecto es idéntico al que podemos observar en las tallas de madera, en las que se ha trabajado con la gubia y no se la lijado posteriormente.

A partir de aquí, las superficies aparecen perfectamente alisadas y modeladas, lo cual tuvo que ser hecho mediante la utilización de lija (o abrasi- vos de grano muy fino) que no han dejado huella alguna.

\section{El uso del taladro.}

En cinco fragmentos que pertenecen en todos los casos a restos de patas animales o brazos/piernas humanas (fragmentos todos ellos muy pequeños e inidentificables, salvo los dos que reproduce G. Navarrete en su Catálogo de 1987, Lám. 208, arriba) se documenta con precisión el uso de un taladro de $1 \mathrm{~cm}$. de anchura que ha dejado en la piedra un cilindro perfectamente circular y cuyas paredes están perfectamente pulidas, lo que denota una velocidad de giro del taladro muy alta y regular. Dado que es la primera vez que se documenta el uso de este utensilio entre los iberos, y que hasta la fecha carecemos tanto de ellos cuanto de sus representaciones, hemos de referirnos a la imagen de taladros que tenemos a través de las esculturas orientales (egipcia y griega).

\section{Casos específicos.}

Existen dos casos específicos:

- El primero es el de las perforaciones. El caso más logrado es el de los orificios nasales de la nueva cabeza de cordero, en los cuales se ha perforado la piedra en una profundidad de unos $2 \mathrm{~cm}$., consiguiendo posteriormente alisar muy cuidadosamente las superficies interiores de dichas perforaciones.
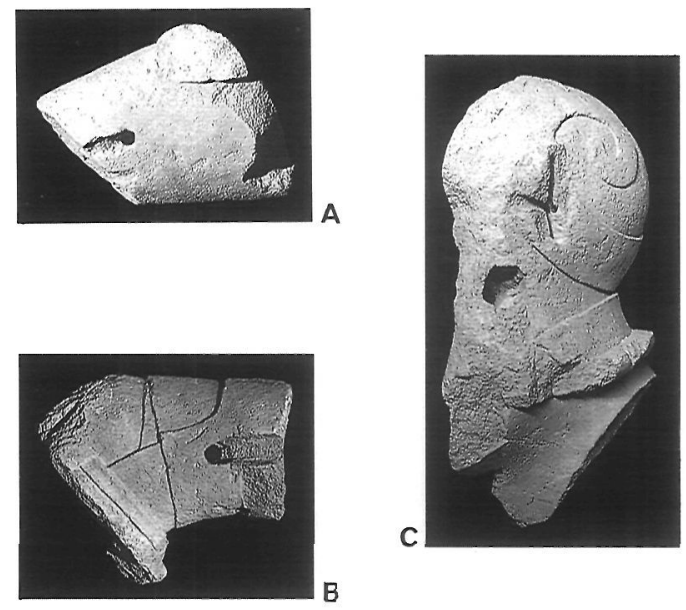$$
8
$$

Lám. 3.- A y B: fragmentos con taladro; C: casco con perforación a golpes y taladro. 
Por el contrario, en todas las demás perforaciones (los cascos, el brazo derecho del guerrero número 2, la gran perforación en la carrillera del casco del mismo guerrero, diversos fragmentos de piernas, la mano derecha del jinete desmontado...) las superficies interiores muestran en sus paredes las irregulares y geométricas huellas de un cincel más o menos estrecho con el que el escultor fué pacientemente perforando.

- El segundo caso específico que merece ser comentado es el de las veladuras o transparencias de partes del cuerpo u objetos situados detrás de la superficie visible de la escultura: la oreja de la cabeza con tocado, que se "transparenta" a través del dicho tocado; el tórax del Varón con Manípulo que se dibuja suavemente bajo la túnica; las fibulas anulares hispánicas que se transparentan bajo sendos ropajes en dos fragmentos desgraciadamente sueltos; el "broche" que asoma bajo la túnica de la Dama con Niño, junto a su escote... Estos ejemplos, de los que los mejores son, sin duda, el Varón con Manípulo y una de las dos fíbulas anulares citadas fueron realizados mediante un empleo muy cuidadoso de la talla y el lijado final.

El escultor que ha tratado estas obras no solamente tenía muy claro que debajo de un ropaje hay un cuerpo articulado o que un vestido se superpone a determinados objetos pero no los oculta, sino que conocía la manera de realizarlo escultóricamente.

Si ponemos todo esto en orden de trabajo, el proceso resultante es bastante lógico: una vez cortado el bloque (lo cual desconocemos como se haría) se iba desbastando la figura con un cincel de boca ancha $(4 \mathrm{~cm}$.) que, logicamente, tuvo que ser golpeado con un martillo. A continuación, con otros cinceles progresivamente más estrechos, según las necesidades concretas de cada parte de la obra, se seguía esta labor de desbaste hasta ir dejando la escultura completamente diseñada y a falta del tratamiento final. Si las superficies a conseguir eran curvas, específicamente cóncavas, se utilizaba un cincel fino ( 9 milímetros) de filo curvo; y si eran rectas o convexas se hacía lo mismo con un cincel de filo recto, de tal manera que aplicando numerosos golpes y muy juntos, prácticamente yuxtapuestos, se conseguía una superficie cuyos rasgos principales ya eran los de la estatua deseada.

Luego, con sumo cuidado, se iba igualando esta superficie con una talla alargada, que hemos des- crito "como si hubiera sido hecha con el filo de un cuchillo" o con una gubía de tallista. Hasta este punto se había trabajado con golpes cortos, secos, y con el cincel incidiendo sobre la superficie de la piedra en un ángulo que debía oscilar entre los 30 y 90 grados. Sin embargo, esta operación descrita se efectuaba de tal manera que el filo del cincel fuese deslizándose sobre la superficie de la obra, con el objeto de alisar el rugoso aspecto que ofrecía.

Una vez que se había alcanzado ese estado de la obra, el aspecto de la superficie era el de una sucesión de alargados planos irregulares yuxtapuestos entre sí mediante aristas sinuosas, aristas que de lejos no se aprecian y que ya daban a la obra el redondeado aspecto casi final.

Entonces quedaba rematar la escultura, lo cual se hacía aunque no tenemos huellas del método usado, mediante el meticuloso lijado de las superficies. No puede decirse si para ello se utilizó la lima o directamente abrasivos minerales en polvo, pues no ha quedado huella de ello perceptible a simple vista. Pero, en todo caso, es cierto que la técnica de lijado y pulimento utilizada era muy desarrollada a juzgar por las finas gradaciones del modelado que presentan numerosas partes de estas obras.

En algunos casos se conserva como toque final, el testimonio de una capa de pintura roja cubriendo la superficie de la piedra. Se trata de una capa muy ligera y apenas consistente. Se localiza sobre los cuerpos de animales y en algunas prendas de cuero de las personas.

Según todo ello, nos es posible reconstruir una tabla mínima de los instrumentos que fueron utilizados, que se ofrece en la figura adjunta.

En el caso de la estatuaria helena las técnicas utilizadas son relativamente conocidas. Adams analiza detenidamente (2) todo el proceso desde el Arcaismo hasta el Helenismo, resumiendo y actualizando los anteriores y clásicos trabajos de Blümel (3) y Casson (4). La tabla de instrumentos que la investigación acepta actualmente que fueron usados

(2) S. ADAMS: The Technique of Greek Sculpture. London, 1967.

(3) C. BLÜMEL: Griechische Bildhauerarbeit. Berlin y Lepzig, 1927. Redd. IDEM: Griechische Bildhauer an der Arbeit, W. de Gruyter, Berlin, 1953 edición inglesa en 1955.

(4) S. CASSON: The Technique of Early Greek Sculpture, New York, 1970 (1. ${ }^{\text {a }}$ ed. en 1933). 
por los talleres griegos no es muy diferente de la que nosotros ofrecemos aquí para los talleres ibéricos de Porcuna. Se incluye entre aquellos el puntero y la lima como instrumentos más importantes de entre los que nosotros no tenemos documentados, pero en lo sustantivo las tareas principales del trabajo fueron, al parecer, realizadas de modo muy similar.
Adams recoge como principales abrasivos utilizados por los griegos el esmeril (natural del Cabo Emeri, en la isla griega de Naxos), la pumita (piedra pómez) y el corindón. Una vez suficientemente machacados y reducidos a polvo, se aplicaban pacientemente por frotación contra la superficie de la obra escultórica. 